ARTICLE

\title{
Anomalous mechanical materials squeezing three-dimensional volume compressibility into one dimension
}

\author{
Xingxing Jiang1,2, Maxim S. Molokeev³,4, Liyuan Dong6 ${ }^{6}$, Zhichao Dong7, Naizheng Wang (1) 1, Lei Kang ${ }^{1,2}$,

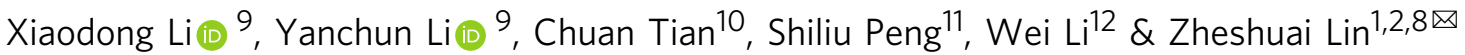

\begin{abstract}
Anomalous mechanical materials, with counterintuitive stress-strain responding behaviors, have emerged as novel type of functional materials with highly enhanced performances. Here we demonstrate that the materials with coexisting negative, zero and positive linear compressibilities can squeeze three-dimensional volume compressibility into one dimension, and provide a general and effective way to precisely stabilize the transmission processes under high pressure. We propose a "corrugated-graphite-like" structural model and discover lithium metaborate $\left(\mathrm{LiBO}_{2}\right)$ to be the first material with such a mechanical behavior. The capability to keep the flux density stability under pressure in $\mathrm{LiBO}_{2}$ is at least two orders higher than that in conventional materials. Our study opens a way to the design and search of ultrastable transmission materials under extreme conditions.
\end{abstract}

\footnotetext{
${ }^{1}$ Technical Institute of Physics and Chemistry, Chinese Academy of Sciences, Beijing 100190, China. ${ }^{2}$ Center of Materials Science and Optoelectronics Engineering, University of Chinese Academy of Sciences, Beijing 100049, P.R. China. ${ }^{3}$ Laboratory of Crystal Physics, Kirensky Institute of Physics, SB RAS, Krasnoyarsk 660036, Russia. ${ }^{4}$ Department of Physics, Far Eastern State Transport University, Khabarovsk 680021, Russia. ${ }^{5}$ Siberian Federal University, Krasnoyarsk 660041, Russia. ${ }^{6}$ Wuhan National Laboratory for Optoelectronics and School of Physics, Huazhong University of Science and Technology, Wuhan 430074, China. ${ }^{7}$ Laboratory of Space Astronomy and Technology, National Astronomical Observatories, Chinese Academy of Sciences, Beijing 100101, China. ${ }^{8}$ University of Chinese Academy of Sciences, Beijing 100049, China. ${ }^{9}$ Beijing Synchrotron Radiation Facility, Institute of High Energy Physics, Chinese Academy of Sciences, Beijing 100049, China. ${ }^{10}$ Institute of Deep-sea Science and Engineering, Chinese Academy of Sciences, Sanya 572000, China. ${ }^{11}$ Institute of Mechanics, Chinese Academy of Sciences, Beijing 100190, China. ${ }^{2}$ School of Materials Science and Engineering; TKL of Metal and MoleculeBased Material Chemistry, Nankai University, Tianjin 300350, China. ${ }^{凶}$ email: zslin@mail.ipc.ac.cn
} 
T he anomalous mechanical materials provide a novel approach to modulate the functional properties that is inaccessible by conventional materials, owing to their counterintuitive squeeze-expanded ${ }^{1}$, stretch-densified ${ }^{2}$, push/ pull-twisted $^{3}$, or negative-modulus ${ }^{4}$ response. In particular, the control of compressibilities in these materials can precisely regulate the physical properties under high-pressure conditions, which shows wide application prospects in, e.g., superconductivity modulation ${ }^{5}$, ferroelectricity enhancement ${ }^{6}$, ultrasensitive sensing ${ }^{7}$, ultrastable information communication ${ }^{2,8}$, energy harvesting ${ }^{9}$, seismic wave detection ${ }^{1,10}$, and body armor fabrication ${ }^{11,12}$. As many physical properties are tightly related to the transmission processes of electrons, photons, and phonons ${ }^{13}$, the transmission stability under pressure is essential to improve the performances of functional materials in extreme and complex environments, but the relevant studies in anomalous mechanical materials have been elusive.

In a transmission process, the energy or particle flux density is determined by the transmission power and the cross section vertical to the propagating direction in the material ${ }^{14}$. In order to stabilize the transmission processes under pressure, the cross section, normally contracted with the increase of ambient pressure, needs to be kept as constant as possible to resist the influence of pressure fluctuation $\Delta P$. To this end, a most effective approach is to make the linear compressibility $\left(\alpha_{l}=-(1 / l)(\Delta l / \Delta P)\right)$ along the propagating direction $\hat{l}$ exactly equal to the volume compressibility $\left(\alpha_{V}=\right.$ $-(1 / V)(\Delta V / \Delta P), \mathrm{V}$ is volume), i.e., to squeeze the three-dimensional volume compressibility into one dimension. Under this condition, the area compressibility on the cross section perpendicular to the $\hat{l}$ direction is kept zero under pressure so as to remove the influence of $\Delta P$, considering that the volume compressibility is identically equal to the sum of linear compressibilities for any three orthogonal axes $^{15}$. Note that for a specific material volume compressibility is a constant which can be represented by a scalar without directivity, while linear compressibility is a "vector" depending on direction. The "vectorization" would endow volume compressibility with the "directionality" to subtly manipulate physical properties. However, this mechanical matching condition is impossible to be realized in conventional materials which are shrinked along arbitrary axis under pressure (i.e., exhibiting positive linear compressibility, or PLC, behavior in arbitrary direction), since the volume compressibility is always larger than the linear compressibility along any direction in these materials. This problem can be solved in anomalous mechanical materials with negative linear compressibility (NLC) and/or zero linear compressibility (ZLC).

NLC and ZLC materials possess the pressure-responding behavior that the size along a specific direction expands ${ }^{2,16}$ and keeps constant ${ }^{17}$, respectively, when being squeezed by hydrostatic pressure. These counterintuitive pressure-responding behaviors violate the commonly well-recognized "compressioncontraction" effect, and have promising applications in many advanced technologies, e.g., in ultra-sensitive pressure sensors ${ }^{2}$, deep-sea optical communications ${ }^{8}$ and smart body armors ${ }^{12}$. After some mathematical deductions (see Sect. S1), we conclude that: (i) when an NLC axis is introduced into the normal mechanical system, the condition for squeezing threedimensional volume compressibility into one dimension, i.e., the exact matching between volume and linear compressibilities, can be achieved only if the absolute value of NLC coefficient is larger than either of the PLC coefficients. Nevertheless, this matching condition is seldom satisfied in practice, because in almost all known NLC materials the NLC coefficient is smaller than the PLC values. (ii) when a ZLC axis is introduced, the matching condition cannot be satisfied as well. (iii) when PLC, NLC, and ZLC are independently coexisted in the system, the matching direction can be always found.
However, both NLC and ZLC were separately discovered only in a handful of materials ${ }^{2,17}$ and the coexistence of NLC, ZLC, and PLC has never been demonstrated in any material. From the viewpoint of molecular geometry, the NLC behavior frequently occurs in the one-dimensional chains or two-dimensional layers with wrinkles, which can be flattened by the extrusion of neighboring counterparts to generate the squeeze-expanded behavior under pressure, as summarized in the famous Lifshitz mechanism 18,19 . On the other hand, the ZLC behavior has been observed in the graphite structure with the flat and strongly covalent two-dimensional layers constructed by the threecoordinated carbon atoms, which almost keeps constant up to the phase-transition pressure with the linear compressibility along the a(b)-axis almost equals to that of diamond ${ }^{20}$. Inspiring from these studies, we speculate that, if the graphite layers become corrugated along a specific axis, the wrinkles in layers would be flattened by the extrusion of neighbor layers, and thus the NLC can be induced along this direction. The corrugatedgraphite-like (CGL) architecture at a molecular level, therefore, would achieve the coexistence of NLC, ZLC, and PLC in a single material.

Analogous to carbon atoms in graphite, boron atoms can be three-coordinated with oxygen to form the strongly covalent $\left[\mathrm{BO}_{3}\right]$ groups. The planar $\left[\mathrm{BO}_{3}\right]$ groups are likely to further connect with other groups to form infinite two-dimensional layers $^{21,22}$, by which the anomalous mechanical properties have been observed, e.g., in $\mathrm{KBe}_{2} \mathrm{BO}_{3} \mathrm{~F}_{2}{ }^{23}$ and in $\mathrm{AEB}_{2} \mathrm{O}_{4}{ }^{17}$.

In this work, by applying the CGL model to borates, we identify that lithium metaborate $\left(\mathrm{LiBO}_{2}\right)$ possesses the simultaneous PLC, ZLC, and NLC behaviors from 0 to $2.52 \mathrm{GPa}$, so the volume compressibility can be squeezed into one dimension. The capability of flux density stability in $\mathrm{LiBO}_{2}$ is higher by at least two orders than conventional materials. Meanwhile, $\mathrm{LiBO}_{2}$ exhibits a good optical transparency from deep-ultraviolet (DUV) to near-infrared (IR) region, manifesting its promising prospect for ultrastable optical transmission in high-pressure environments.

\section{Results}

Crystal structure. Our refined structure analysis reveals that $\mathrm{LiBO}_{2}$ crystalizes in monoclinic $\mathrm{P}_{1} / \mathrm{c}$ space group at ambient pressure, in which boron and lithium atoms are threefold and fourfold coordinated with oxygen atoms to form $\left[\mathrm{BO}_{3}\right]$ triangles and $\left[\mathrm{LiO}_{4}\right]$ triangular pyramids, respectively, as firstly determined by Zachariasen ${ }^{24}$. By sharing the corner oxygen atoms, the $\left[\mathrm{BO}_{3}\right]$ triangles are connected with each other, giving rise to an infinite $\left[\mathrm{BO}_{2}\right]_{\infty}$ belt along the b-axis (Fig. 1a). The $\left[\mathrm{BO}_{2}\right]_{\infty}$ belts are bounded by the $\left(\mathrm{LiO}_{3}\right)$ bases in $\left[\mathrm{LiO}_{4}\right]$ triangular pyramids along the a-axis to construct the $\left[\mathrm{LiBO}_{2}\right]_{\infty}$ layer, in which the hexatomic $\left[\mathrm{Li}_{2} \mathrm{BO}_{3}\right]$ and $\left[\mathrm{LiB}_{2} \mathrm{O}_{3}\right]$ rings are alternatively connected to form a graphite-like-layered architecture. The $\left[\mathrm{LiBO}_{2}\right]_{\infty}$ layers are further adhered to one another along the $\mathrm{c}$-axis by the $\mathrm{Li}-\mathrm{O}$ bonds out of the $\left(\mathrm{LiO}_{3}\right)$ bases (Fig. 1b). Note that the introduction of nonplanar $\left(\mathrm{LiO}_{3}\right)$ bases produces wrinkles along the a-axis in the $\left[\mathrm{LiBO}_{2}\right]_{\infty}$ layer, and thus forming a typical CGL structure.

Coexistence of NLC, ZLC, and PLC. The high-pressure XRD demonstrates that the single phase of $\mathrm{LiBO}_{2}$ is well preserved from 0 to $2.52 \mathrm{GPa}$, as no new XRD peak emerges or vanishes (Fig. 1c and Fig. S2). The extracted cell parameters (Fig. 1d) reveal that the crystallographic a-axis is anomalously elongated by about $1 \%$ in the measured pressure range, indicating the NLC behavior along this direction, while the b-axis remains nearly constant and only contracts by $0.4 \%$. In comparison, the c-axis experiences very prominent shrinkage of $3.1 \%$. The corresponding 

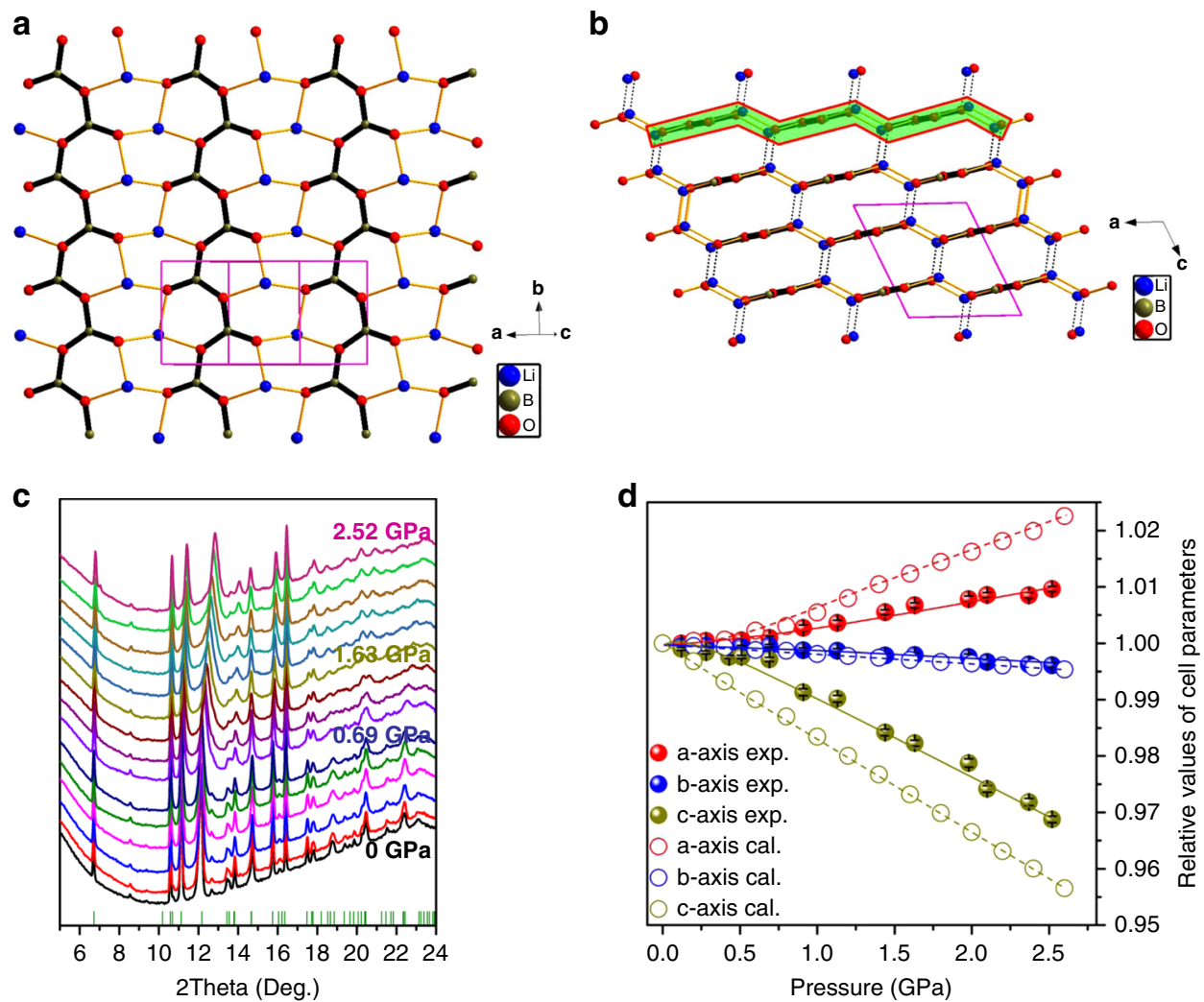

Fig. 1 Crystal structure and linear compressive behaviors in $\mathbf{L i B O}_{2} \cdot \mathbf{a}$ Graphite-like $\left[\mathrm{LiBO}_{2}\right]_{\infty}$ layer (in the a-b plane), in which the $\left[\mathrm{BO}_{2}\right]_{\infty}$ belt is highlighted by thick bars in a zig-zag configuration. b Bond connection between the neighbor $\left[\mathrm{LiBO} \mathrm{B}_{\infty}\right]_{\infty}$ layers (along the c-axis), in which the corrugations introduced by $\left(\mathrm{LiO}_{3}\right)$ bases are highlighted by green strips. The unit cells are represented by pink boxes. c evolution of the high-pressure XRD patterns. d Variations of $a$-axis, $b$-axis, and $c$-axis with respect to hydrostatics pressure. Experimental and first-principles results are represented by solid and open circles, respectively.

linear compressibilities along the mechanical principal $X$-, $Y$ - and $Z$ axes are $-5.33(34) / \mathrm{TPa}, 1.66(11) / \mathrm{TPa}$, and 25.63(97)/TPa, respectively (The transformation matrix between crystallographic and mechanical principal axes see Table S3). Note that the compressibility along the $\mathrm{Y}$-axis is much smaller than that in the majority of materials and can be categorized into ZLC ${ }^{25,26}$. Therefore, the NLC, ZLC, and PLC are simultaneously presented in $\mathrm{LiBO}_{2}$. In principle, one may always find the ZLC direction in an NLC material by determining the specific direction between NLC and PLC axes. However, the existence of NLC, PLC, and ZLC axes in $\mathrm{LiBO}_{2}$ is entirely independent of each other.

Mechanism elucidation. To shed light on the microscopic mechanical mechanism in $\mathrm{LiBO}_{2}$, the computational simulations were performed. The finite element analysis (FEA) reveals that most of the stress under pressure is undertaken by $\left[\mathrm{BO}_{3}\right]$ triangles (Fig. 2a), indicating that the quasi-rigid $\left[\mathrm{BO}_{2}\right]_{\infty}$ belts play the key role to resist the external pressure. In comparison, the strain is concentrated on $\left[\mathrm{LiO}_{4}\right]$ triangular pyramids (Fig. 2b), demonstrating that the deformation in these microscopic units dominantly determine the pressure-induced structural modification in $\mathrm{LiBO}_{2}$.

Because the light lithium and boron atoms have low X-ray scattering factor, it is very difficult to accurately determine their atomic position variation by high-pressure XRD. In order to overcome this problem, the first-principles simulations with high precision were adopted. The calculated compressibilities along the $X$-, $Y$ - and $Z$-axes are $-14.50(51) / \mathrm{TPa}, 1.77(01) / \mathrm{TPa}$, and 38.47 (70)/TPa, respectively (Fig. $1 \mathrm{~d}$ and Table S2), in well consistence with the experimental values. The atomic simulations (see
Table S4) reveal that, from 0 to $2.52 \mathrm{GPa}$, the average $\mathrm{B}-\mathrm{O}$ bond length and $\angle \mathrm{O}-\mathrm{B}-\mathrm{O}$ angle in the $\left[\mathrm{LiBO}_{2}\right]_{\infty}$ layer only decrease by $0.1 \%$ and $0.2 \%$, respectively (Fig. $2 \mathrm{c}, \mathrm{e}$ ), confirming the large rigidity of the $\left[\mathrm{BO}_{2}\right]_{\infty}$ chains (along the $Y$ - or the $b$-axis) formed by $\left[\mathrm{BO}_{3}\right]$ triangles. This makes $\mathrm{LiBO}_{2}$ exhibit a $\mathrm{ZLC}$ behavior along this direction, similar to the role of carbon triangles to ZLC in graphite. Since the covalent strength of $\mathrm{B}-\mathrm{O}$ bond is smaller than that of $\mathrm{C}-\mathrm{C}$ bond, the $\mathrm{ZLC}$ effect in $\mathrm{LiBO}_{2}$ is weaker than that in graphite $(\sim 0.7 / \mathrm{TPa})^{20}$ and diamond $(0.75 / \mathrm{TPa})^{27}$. Owing to the rigidity of $\left[\mathrm{BO}_{2}\right]_{\infty}$ chains, the mechanical strain principally occurs at the $\left[\mathrm{LiO}_{4}\right]$ triangular pyramids, and thus results in different mechanical behaviors along the $X$ - (nearly the $a$-) and $Z$ axes: On one hand, the $\mathrm{Li}-\mathrm{O}$ bonds protruding outside the $\left[\mathrm{LiBO}_{2}\right]_{\infty}$ layers are significantly contracted by $\sim 1.0 \%$ (Fig. $2 \mathrm{c}$ ), which gives rise to the normal PLC along the $Z$-axis. On the other hand, the vertical stress from the approaching of neighbor $\left[\mathrm{LiBO}_{2}\right]_{\infty}$ layers makes the $\left[\mathrm{LiO}_{3}\right]$ bases (i.e., the wrinkles in the CGL architecture) flatten with the $\angle \mathrm{O}-\mathrm{Li}-\mathrm{O}$ angles dramatically opened by $3.8 \%$ (Fig. 2d). This results in the expansion of $\left[\mathrm{LiBO}_{2}\right]_{\infty}$ layers in response to the increase of hydrostatic pressure, which transcends the contraction of intralayer $\mathrm{Li}-\mathrm{O}$ bonds $(\sim 1.0 \%)$ caused by the in-plane strain, and eventually give rise to the net NLC behavior along the $X$-axis (Fig. 2f). Therefore, the corrugated $\left[\mathrm{LiBO}_{2}\right]_{\infty}$ layers with CGL architecture is the structural origin for the simultaneous occurrence of NLC, ZLC, and PLC in $\mathrm{LiBO}_{2}$, as depicted in Fig. $2 \mathrm{~g}$.

Application prospects from mechanical properties. Figure $3 \mathrm{a}$ plots the exact curve squeezing three-dimensional volume compressibility into one dimension in $\mathrm{LiBO}_{2}$. In comparison, owing 
a

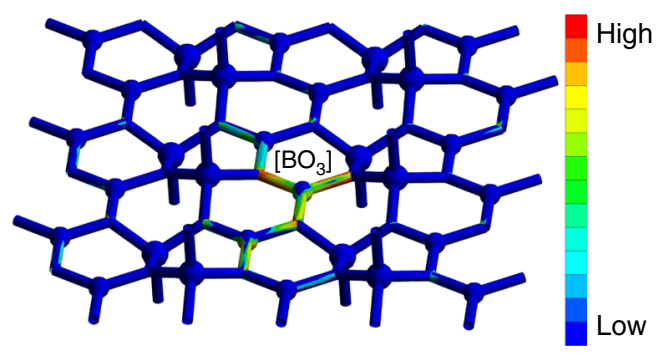

b

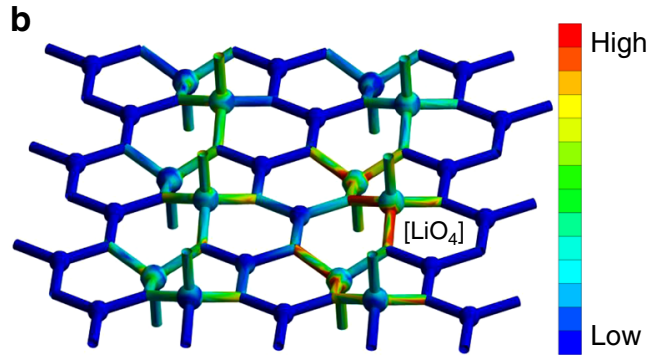

C

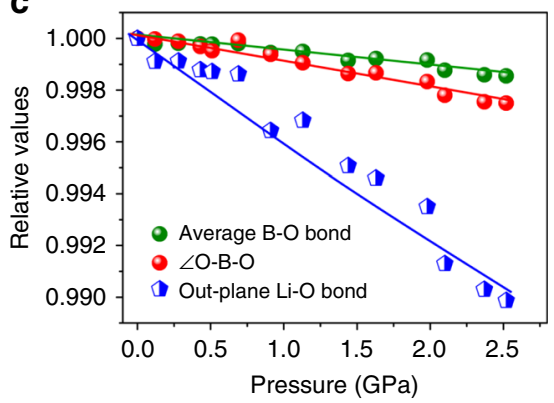

e

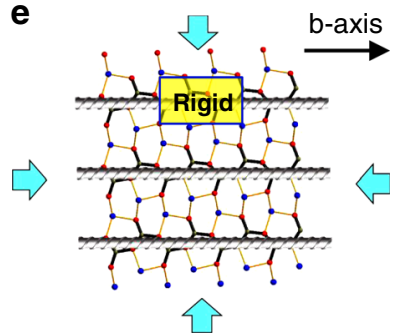

d 1.05

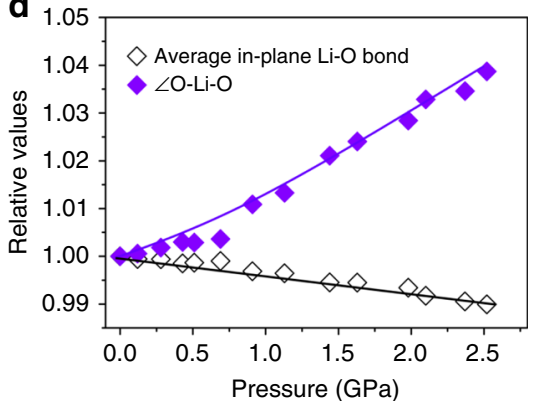

$\mathbf{g}$

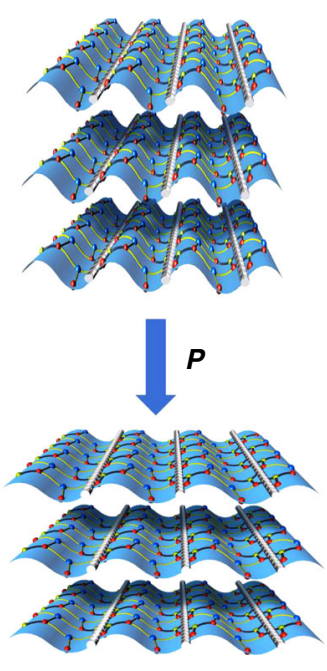

Fig. 2 Microscopic mechanism for the simultaneous NLC, ZLC, and PLC in $\mathbf{L i B O}_{\mathbf{2}}$. a Stress and $\mathbf{b}$ Strain distribution in $\mathrm{LiBO}_{2}$ structure, respectively, by finite element analysis. c, d Pressure-induced modification of bond lengths and bond angles responsible for ZLC and NLC. e Schematic for the ZLC behavior. $\mathbf{f}$ Schematic for the NLC behavior. $\mathbf{g}$ Schematic for the mechanism of the pressure-responding behaviors originated from the CGL structure. The $\left[\mathrm{BO}_{2}\right]_{\infty}$ chains are represented by gray bars and the $\left[\mathrm{LiO}_{3}\right]$ bases are represented by blue corrugations.

to the large discrepancy between volume and linear compressibilities the matching curves do not exist in $\mathrm{Ag}_{3} \mathrm{Co}(\mathrm{CN})_{6}$ (the material with largest NLC) ${ }^{8}$, diamond ${ }^{27}$ and graphite ${ }^{20}$ (the ZLC materials), and the most used transmission materials, e.g., copper $^{28}$ and quartz ${ }^{29}$ (Fig. S5). This indicates that $\mathrm{LiBO}_{2}$ has the superior capability to alleviate the influence of pressure on the flux density stability compared with other materials. Taking the deep-sea environment as an example, when moved from the sea level to the Mariana Trench (the deepest trench on earth with the depth of $\sim 11,000 \mathrm{~m}$ ), the relative fluctuations of flux density in $\mathrm{Ag}_{3} \mathrm{Co}(\mathrm{CN})_{6}$, diamond, graphite, copper, and quartz are at least two orders larger than that in $\mathrm{LiBO}_{2}$ (Fig. 3b), on the condition that the resolution for the compressibility-angle-tuning is one degree (see the detailed discussion in Tables S5 and S6). Therefore, $\mathrm{LiBO}_{2}$ can bring out great advantage in the ultrastable transmission apparatus in high pressure-fluctuating environments. Moreover, the UV-Vis diffuse-reflectance spectrum reveals that $\mathrm{LiBO}_{2}$ has very good optical transparency in the range from DUV to near-IR (Fig. 3c). The further first-principles calculations show that the UV absorption edge of $\mathrm{LiBO}_{2}(175 \mathrm{~nm}$ under atmospheric pressure) is blue-shifted as pressure increases (inset in Fig. $3 \mathrm{c}$ and Table S7), demonstrating that the wide optical transmittance in $\mathrm{LiBO}_{2}$ is well kept under pressure. Therefore, from a proof-of-principle perspective $\mathrm{LiBO}_{2}$ exhibits a promising prospect to be used in the ultra-precise optical detection apparatus in high pressure-fluctuating environments, e.g., in deep-sea, as schematically depicted in Fig. 3d. Independent of the deep-sea pressure, the optical cross-section area in $\mathrm{LiBO}_{2}$ can almost keep constant, and thus the fluctuation of optical flux density owing to the ambient pressure is eliminated and the signal from detection target is transmitted with ultra-high fidelity. Doubtlessly, to realize these practical applications, there is still a long way to go for overcoming lots of technical hurdles, such as large-size single crystal growth and water-proof treatment, which, however, we believe can be eventually solved with the development of advanced techniques.

\section{Discussion}

Aiming at stabilizing flux density under pressure, we have exhibited a rational materials design from physical and mathematical derivation, structural model proposition to material discovery, and $\mathrm{LiBO}_{2}$ was discovered as the first material, to the best of our knowledge, with coexisting NLC, ZLC, and PLC to "vectorize" volume compressibility into the linear compressibility in a specific direction. By virtue of this fascinating mechanical property in combination with excellent optical transparency, $\mathrm{LiBO}_{2}$ holds great promise in the ultrastable optical apparatus under high pressure-fluctuating conditions. More importantly, both pressure and volume are involved in the equation of state and determine the basic thermodynamic functions of state (e.g., enthalpy and free energy). The "vectorization" of volume compressibility, the physical quantity directly connecting these twostate parameters, would greatly improve the tuning ability of pressure on the functional properties and may provide a freedom to modulate the performance of materials related to the 
a

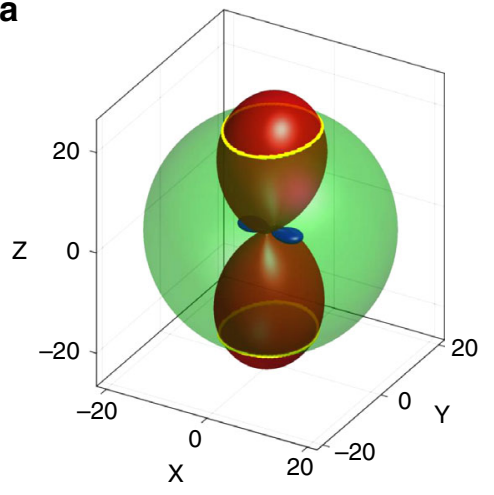

b

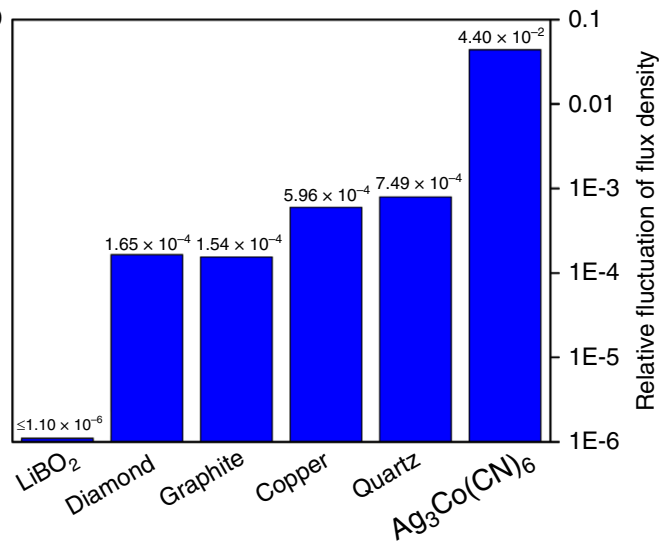

d
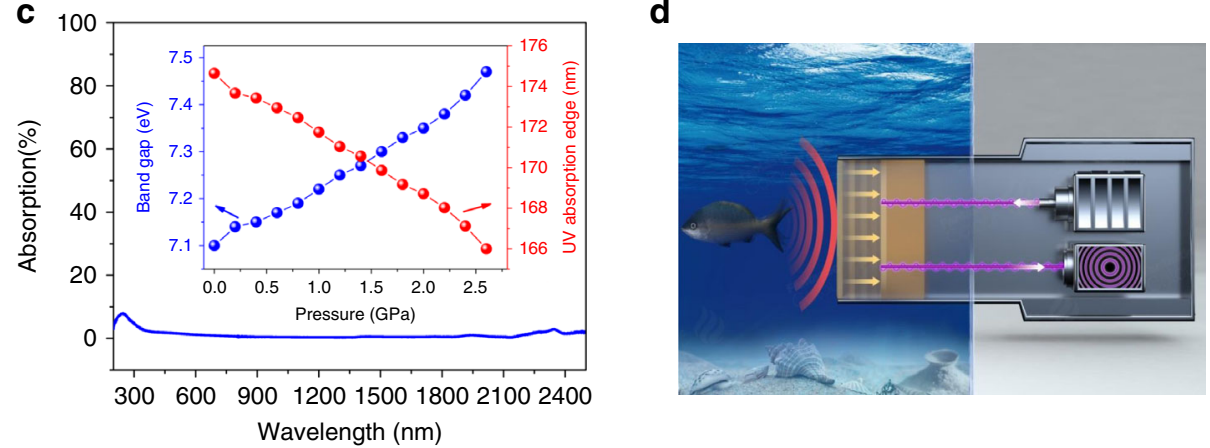

Fig. 3 Flux density stability, optical property, and potential application of $\mathbf{L i B O}_{\mathbf{2}}$. a The matching between linear and volume compressibilities. Positive and negative linear compressibilities are represented by red and blue surfaces, and volume compressibility is represented by green sphere, respectively. The isoline between linear and volume compressibilities is highlighted by yellow curve. $\mathbf{b}$ Comparison of the relative fluctuations of flux density among $\mathrm{LiBO}_{2}$ and other materials, as they are moved from the sea level to Mariana Trench. $\mathbf{c} U V$-Vis diffuse-reflectance spectrum. The calculated dependence of pressure vs. UV absorption edge (i.e., band gap) is shown in the inset. $\mathbf{d}$ Schematic for the ultrastable optical detection apparatus fabricated by $\mathrm{LiBO}_{2}$ in the deep-sea environment.

transmission of electrons, photons and phonons, such as electric conductivity, optical waveguide, and thermal conductivity.

\section{Methods}

Sample preparation. Polycrystalline $\mathrm{LiBO}_{2}$ was synthesized through solid-state reaction in stoichiometric ratio with analytically pure $\mathrm{Li}_{2} \mathrm{CO}_{3}(99.99 \%)$ and $\mathrm{B}_{2} \mathrm{O}_{3}$ (99.98\%) purchased from Sigma-Aldrich corporation as the starting materials. These ingredients were mixed homogeneously, then heated with the heating rate $30^{\circ} \mathrm{C} / \mathrm{h}$ in a muffle furnace. The ingredients were taken out and carefully grinded at $200{ }^{\circ} \mathrm{C}, 400{ }^{\circ} \mathrm{C}$, and $600{ }^{\circ} \mathrm{C}$. Finally, the temperature was raised to $700{ }^{\circ} \mathrm{C}$ and kept for $24 \mathrm{~h}$. After cooling to room temperature, the white powder of target compound was obtained.

High-pressure X-ray diffraction. The high-pressure XRD experiment was conducted in the 4W2 beam line with the radiation at $0.6199 \AA$ of Beijing Synchrotron Radiation Facility (BSRF). The X-ray beam at the wavelength $0.6199 \AA$ was focused into a $36 \times 12 \mu \mathrm{m}^{2}$ spot using Kirkpatrick-Baez mirrors. The hydrostatic pressure was exerted by the systematic diamond anvil cells (DAC) with the culet diameter of $400 \mu \mathrm{m}$. The sample in well grinded powder was placed in a hole of about $120 \mu \mathrm{m}$ diameter in a pre-indented stainless steel gasket with the thickness of $40 \mu \mathrm{m}$. Silicon oil was adopted to act as the pressure-transmitting medium and ruby chips were placed for pressure calibration by measuring the fluorescence shift as a function of pressure $^{30}$. The diffraction patterns were recorded by a Pilatus image plate and integrated with the FIT2D software package. The pressure-depending lattice parameters were refined by Rietveld refinement ${ }^{31}$ using TOPAS software ${ }^{32}$. The compressibility were fitted by the PASCal software ${ }^{33}$.

The determination of cell parameters under high pressure is influenced by the XRD peak-broadening due to the pressure inhomogeneity from pressuretransmitting medium. It is well known that the hydrostatic-pressure limit of silicon oil is about $0.9 \mathrm{GPa}^{34}$, which is lower than the upper-pressure limit of $2.52 \mathrm{GPa}$ in this work. At the first glance, the use of silicon oil would deteriorate the accuracy of the refined cell parameters. However, as displayed in Table S1, the broadness of peaks just affects the third decimal place (i.e., in the magnitude of $0.003 \AA$ ), although the error of the cell parameters is increased by about three times (taking the $a$-axis as an example, from 0.0009 to $0.0027 \AA$ ) when pressure increases from 0 to $2.52 \mathrm{GPa}$. In comparison, the pressure-induced change of the cell parameters is in the magnitude of $0.02 \AA$, one-order higher than the influence of the error. Therefore, the peak-widening due to the pressure inhomogeneity from silicon oil does not affect the conclusion that NLC, ZLC, and PLC behaviors are coexisted in $\mathrm{LiBO}_{2}$.

Raman spectrum. The Raman pattern was recorded from 100 to $2000 \mathrm{~cm}^{-1}$ at room temperature, using in Via-Reflex, equipped with a solid-state laser with a wavelength of $532 \mathrm{~nm}$. In order to improve the signal to noise ratio of the spectrum, 10 integrations were carried out with an integration time of $10 \mathrm{~s}$ at a resolution of $1 \mathrm{~cm}^{-1}$.

Infrared spectrum. The infrared spectrum was obtained at room temperature in a range from 400 to $2000 \mathrm{~cm}^{-1}$ with resolution of $1 \mathrm{~cm}^{-1}$ via a Bio-Rad FTS-60 FTIR spectrometer. The sample and dried $\mathrm{KBr}$ ( $5 \mathrm{mg}$ of the sample, $500 \mathrm{mg}$ of $\mathrm{KBr}$ ) were mixed thoroughly together.

UV-Vis Diffuse Reflectance Spectroscopy. The diffuse reflectance data were collected with a Varian Cary 7000 UV-vis-NIR spectrometer equipped with an integrating sphere in the wavelength range from 200 to $2500 \mathrm{~nm} . \mathrm{BaSO}_{4}$ was employed as the $100 \%$ reflectance standard.

Finite element analysis (FEA). FEA was performed by ANSYS Workbench software. The $\left[\mathrm{BO}_{3}\right]$ and $\left[\mathrm{LiO}_{4}\right]$ groups were respectively modeled by four and five rigid balls (modeling the atoms) connected by three and four small bars (modeling the bonds) with the same diameter. Based on the spacial arrangement of the microscopic groups in the crystal lattice, the whole architecture was built by 36 $\left[\mathrm{BO}_{3}\right]$ and $36\left[\mathrm{LiO}_{4}\right]$ units as depicted in Fig. S1. Since the characteristic vibrational frequencies of $\mathrm{B}-\mathrm{O}$ bond in $\left[\mathrm{BO}_{3}\right]$ and $\mathrm{Li}-\mathrm{O}$ in $\left[\mathrm{LiO}_{4}\right]$ groups are about $1500 \mathrm{~cm}^{-1}$ and $400 \mathrm{~cm}^{-1}$ (refs. ${ }^{35,36}$ ), the elastic coefficient ratio between the two types of bonds are about 22:1, owing to the formula $k=m \omega^{2}$, where $k, \omega$, and $m$ are elastic coefficient, vibrational frequency, and atomic mass, respectively. The elastic modulus of the balls (modeling the atoms) was set to $10^{6}$ orders higher than that of B-O bonds in order to manifest their rigidity. The FEA model was meshed into 80758 tetrahdral elements which are connected by 161,243 nodes, and the hydrostatic 
pressures of $2.52 \mathrm{GPa}$ was applied on the surfaces of the model. Finally, by solving the Cauchy continuum mechanics equations, the von-Mises equivalent stress and strain can be retrieved. As periodic boundary condition in the crystal lattice was not considered in the FEA simulation, to eliminate the boundary effect, the stress and strain distributions near center part were focused on to investigate the mechanical mechanism (Fig. S1).

First-principles calculations. First-principles calculation was performed by CASTEP $^{37}$, a plane-wave pseudopotential total energy package based on density functional theory (DFT) ${ }^{38,39}$. The functionals developed by Perdew, Burke, and Ernzerhof $(\mathrm{PBE})^{40}$ in generalized gradient approximation (GGA) ${ }^{41}$ form were adopted to model the exchange-correlation terms in Hamiltonian. The optimized ultrasoft pseudopential ${ }^{42}$ were used to model the effective interaction between the valence electrons and atom cores, which allow us to use a small plane basis set without compromising the accuracy required by the calculation. High kinetic energy cutoff of $300 \mathrm{eV}$ and Monkhorst-pack ${ }^{43} k$-point mesh spanning $<0.03 \AA^{-1}$ in the Brillouin zone were chosen.

The theoretical cell parameters at various pressures were calculated by geometry optimization with both cell parameters and atomic position relaxed under different hydrostatic pressures, in which the Broyden-Fletcher-Goldfarb-Shanno ${ }^{44}$ minimization scheme was used. The convergence criteria for energy, maximum force, maximum stress and maximum displacement were set as $10^{-5} \mathrm{eV} /$ atom, $0.03 \mathrm{eV} /$ angstrom, $0.05 \mathrm{GPa}$, and $0.001 \AA$, respectively. The compressibilities based on the theoretical cell parameters were also fitted by the PASCal software ${ }^{33}$. The constituent atoms in $\mathrm{LiBO}_{2}$ are very light and their atomic scattering factor is very low, especially for lithium. The structural modification induced by pressure cannot be accurately determined by experiments only (see the experimentally refined CIF files in SI). The variation of atomic positions was determined by first-principles geometry optimization with the cell parameters fixed at the experimental values. For the electronic band structure calculation of $\mathrm{LiBO}_{2}$ the hybridized PBE0 functionals ${ }^{45}$ were adopted. Our previous studies have demonstrated that this type of functionals can accurately predict the band gaps of UV and deep-UV borates ${ }^{46,47}$. Raman and infared spectra were calculated by linear response $\operatorname{method}^{48}$. In band gap prediction and Raman/infrared spectrum calculation, norm-conserving pseudopotential ${ }^{49}$ and kinetic energy cutoff of $900 \mathrm{eV}$ were used.

\section{Data availability}

The authors declare that all the data supporting the findings of this study are available from the authors

Received: 22 June 2020; Accepted: 29 September 2020;

Published online: 05 November 2020

\section{References}

1. Nicolaou, Z. G. \& Motter, A. E. Mechanical metamaterials with negative compressibility transitions. Nat. Mater. 11, 608-613 (2012)

2. Baughman, R. H., Stafstrom, S., Cui, C. X. \& Dantas, S. O. Materials with negative compressibilities in one or more dimensions. Science 279, 1522-1524 (1998).

3. Frenzel, T., Kadic, M. \& Wegener, M. Three-dimensional mechanical metamaterials with a twist. Science 358, 1072-1074 (2017).

4. Fang, N. et al. Ultrasonic metamaterials with negative modulus. Nat. Mater. 5, 452-456 (2006)

5. Uhoya, W. et al. Anomalous compressibility effects and superconductivity of $\mathrm{EuFe}_{2} \mathrm{As}_{2}$ under high pressures. J. Phys.: Condens. Matter 22, 292202 (2010).

6. Baughman, R. H. et al. Negative Poisson's ratios for extreme states of matter. Science 288, 2018-2022 (2000).

7. Cairns, A. B. et al. Giant negative linear compressibility in zinc dicyanoaurate. Nat. Mater. 12, 212-216 (2013).

8. Goodwin, A. L., Keen, D. A. \& Tucker, M. G. Large negative linear compressibility of $\mathrm{Ag}_{3} \mathrm{Co}(\mathrm{CN})_{6}$. Proc. Natl Acad. Sci. USA 105, 18708-18713 (2008).

9. Scheibner, C. et al. Odd elasticity. Nat. Phys. https://doi.org/10.1038/s41567020-0795-y (2020).

10. Lakes, R. S., Lee, T., Bersie, A. \& Wang, Y. C. Extreme damping in composite materials with negative-stiffness inclusions. Nature 410, 565-567 (2001).

11. Greaves, G. N., Greer, A. L., Lakes, R. S. \& Rouxel, T. Poisson's ratio and modern materials. Nat. Mater. 10, 823-837 (2011).

12. Cairns, A. B., Thompsonm, A. L., Tucker, M. G., Haines, J. \& Goodwin, A. L. Rational design of materials with extreme negative compressibility: selective soft-mode frustration in $\mathrm{KMn}\left[\mathrm{Ag}(\mathrm{CN})_{2}\right]_{3}$. J. Am. Chem. Soc. 134, 4454-4456 (2012).

13. Marder, M. P. Condensed Matter Physics (Wiley-VCH: New York, 2012).

14. Flux Density-an overview. ScienceDirect Topics (https://www.sciencedirect. com/topics/engineering/flux-density).
15. Nye, J. F. Physical Properties of Crystals (Oxford University Press, Oxford, 1964).

16. Li, W. et al. Negative linear compressibility of a metal-organic framework. $J$. Am. Chem. Soc. 134, 11940-11943 (2012).

17. Jiang, X. et al. Zero linear compressibility in nondense borates with a "Lu-Ban stool"-like structure. Adv. Mater. 30, 1801313 (2018).

18. Lim, T. 2D structures exhibiting negative area compressibility Phys. Status Solidi B 254, 1600682 (2017).

19. Cairns, A. B. \& Goodwin, A. L. Negative linear compressibility. Phys. Chem. Chem. Phys. 17, 20449-20465 (2015).

20. Yagi, T., Utsumi, W., Yamakata, M., Kikegawa, T. \& Shimomura, O. Highpressure in situ X-ray-diffraction study of the phase-transformation from graphite to hexagonal diamond at room-temperature. Phys. Rev. B 46, 6031-6039 (1992).

21. Chen, C. et al. Nonlinear Optical Borate Crystals-Principles and Applications (Wiley-VCH, New York, 2012).

22. Shen, Y., Zhao, S. \& Luo, J. The role of cations in second-order nonlinear optical materials based on pi-conjugated $\left(\mathrm{BO}_{3}\right)^{3-}$ groups. Coord. Chem. Rev. 366, 1-28 (2018)

23. Jiang, $X$. et al. Isotropic Negative area compressibility over large pressure range in potassium beryllium fluoroborate and its potential applications in deep ultraviolet region. Adv. Mater. 27, 4851-4857 (2015).

24. Zachariasen, W. H. Crystal structure of lithium metaborate. Acta Crystallogr 17, 749-751 (1964)

25. Zeng, Q., Wang, K. \& Zou, B. Near zero area compressibility in a perovskitelike metal-organic frameworks $\left[\mathrm{C}\left(\mathrm{NH}_{2}\right)_{3}\right]\left[\mathrm{Cd}(\mathrm{HCOO})_{3}\right]$. ACS Appl. Mater. \&. Interface 10, 23481-23484 (2018).

26. Xie, Y. et al. Designing orthotropic materials for negative or zero compressibility. Int. J. Solids Struct. 51, 4038-4051 (2014).

27. Occelli, F., Loubeyre, P. \& Letoullec, R. Properties of diamond under hydrostatic pressures up to 140 GPa. Nat. Mater. 2, 151-154 (2003).

28. Engineering ToolBox: Tools and Basic Information for Design, Engineering and Construction of Technical Applications (https://www.engineeringtoolbox. com/bulk-modulus-metals-d_1351.html).

29. Hazen, R. M., Finger, L. W., Hemley, R. J. \& Mao, H. K. High-pressure crystalchemistry and amorphization of alpha-quartz. Solid State Commun. 72, 507-511 (1989).

30. Mao, H. K., Xu, J. \& Bell, P. M. Calibration of the ruby pressure gauge to 800 Kbar under quasi-hydrostatic conditions. J. Geophys. Res.:Solid Earth Planets 91, 4673-4676 (1986).

31. Rietveld, H. M. A profile refinement method for nuclear and magnetic structures. J. Appl. Crystallogr. 2, 65-71 (1969).

32. AXS, B. Bruker AXS TOPAS V4: General profile and structure analysis software for powder diffraction data. - User's Manual (Karlsruhe, 2008).

33. Cliffe, M. J. \& Goodwin, A. L. PASCal: a principal axis strain calculator for thermal expansion and compressibility determination. J. Appl. Crystallogr. 45, 1321-1329 (2012)

34. Angel, R. J., Bujak, M., Zhao, J., Gatta, G. D. \& Jacobsen, S. D. Effective hydrostatic limits of pressure media for high-pressure crystallographic studies. J. Appl. Crystallogr. 40, 26-32 (2007).

35. Voronko, Y. K., Gorbachev, A. V., Kudryavtsev, A. B. \& Sobol, A. A. Ramanscattering study of the structure of alkali-metal borate melts rich in $\mathrm{B}_{2} \mathrm{O}_{3}$. Inorg. Mater. 28, 1368-1372 (1992).

36. Chakrabarti, S., Thakur, A. K. \& Biswas, K. Raman and FTIR spectroscopy study of $\mathrm{LiFeTiO}_{4}$ and $\mathrm{Li}_{2} \mathrm{FeTiO}_{4}$. Ionics 22, 2045-2057 (2016).

37. Clark, S. J. et al. First principles methods using CASTEP. Z. Kristallogr 220, 567-570 (2005)

38. Kohn, W. \& Sham, L. J. Self-consistent equations including exchange and correlation effects. Phys. Rev. 140, 1133-1138 (1965).

39. Payne, M. C., Teter, M. P., Allan, D. C., Arias, T. A. \& Joannopoulos, J. D. Iterative minimization techniques for ab initio total-energy calculations molecular-dynamics and conjugate gradients. Rev. Mod. Phys. 64, 1045-1097 (1992).

40. Perdew, J. P., Burke, K. \& Ernzerhof, M. Generalized gradient approximation made simple. Phys. Rev. Lett. 77, 3865-3868 (1996).

41. Perdew, J. P. \& Wang, Y. Pair-distribution function and its coupling-constant average for the spin-polarized electron-gas. Phys. Rev. B 46, 12947-12954 (1992)

42. Sanchezportal, D., Artacho, E. \& Soler, J. M. Projection of plane-wave calculations into atomic orbitals. Solid State Commun. 95, 685-690 (1995).

43. Monkhorst, H. J. \& Pack, J. D. Special points for Brillouin-zone integrations. Phys. Rev. B 13, 5188-5192 (1976).

44. Pfrommer, B. G., Cote, M., Louie, S. G. \& Cohen, M. L. Relaxation of crystals with the quasi-Newton method. J. Comput. Phys. 131, 233-240 (1997).

45. Adamo, C. \& Barone, V. Toward reliable density functional methods without adjustable parameters: The PBE0 model. J. Chem. Phys. 110, 6158-6170 (1999).

46. He, R. et al. Bandgaps in the deep ultraviolet borate crystals: Prediction and improvement. Appl. Phys. Lett. 102, 231904 (2013). 
47. Lin, Z. et al. First-principles materials applications and design of nonlinear optical crystals. J. Phys. D:Appl. Phys. 47, 253001 (2014).

48. Baroni, S., Gironcoli, D. S., Corso, D. A. \& Giannozzi, P. Phonons and related crystal properties from density-functional perturbation theory. Rev. Mod. Phys. 73, 515-562 (2001).

49. Hamann, D. R., Schluter, M. \& Chiang, C. Norm-conserving pseudopotentials. Phys. Rev. Lett. 88, 135701 (2002).

\section{Acknowledgements}

The authors acknowledge Zhuohong Yin for useful discussions and the experimental time provided by the $4 \mathrm{~W} 2$ beam line of Beijing Synchrotron Radiation Facility (BSRF). This work was supported by the National Scientific Foundations of China (Grants $51702330,11974360,51872297,51890864,21975132$, and 21991143), the Youth Innovation Promotion Association in CAS (Grant 2017035 for X.J.), Young Elite Scientist Sponsorship Program by CAST (YESS), and Fujian Institute of Innovation (FJCXY18010201) in CAS.

\section{Author contributions}

X.J. and Z.L. design the research project. X.J. synthesized the powder sample, and performed the first-principles calculation and optical transmittance measurement. X.J. and N.W. carried out the high-pressure XRD. M.S.M. performed the processing and analysis on the XRD data. L.D., C.T., S.P., and W.L. helped in the design of application in deepoptical application. Z.D. performed the finite element analysis. L.K. helped in the firstprinciples calculation. X.L. and Y.L aided in high-pressure XRD experiment. Z.L. supervised the research and wrote the manuscript.

\section{Competing interests}

The authors declare no competing interests.

\section{Additional information}

Supplementary information is available for this paper at https://doi.org/10.1038/s41467020-19219-5.

Correspondence and requests for materials should be addressed to Z.L.

Peer review information Nature Communications thanks Kai Wang and other, anonymous, reviewers for their contributions to the peer review of this work.

Reprints and permission information is available at http://www.nature.com/reprints

Publisher's note Springer Nature remains neutral with regard to jurisdictional claims in published maps and institutional affiliations.

(c) (i) Open Access This article is licensed under a Creative Commons Attribution 4.0 International License, which permits use, sharing, adaptation, distribution and reproduction in any medium or format, as long as you give appropriate credit to the original author(s) and the source, provide a link to the Creative Commons license, and indicate if changes were made. The images or other third party material in this article are included in the article's Creative Commons license, unless indicated otherwise in a credit line to the material. If material is not included in the article's Creative Commons license and your intended use is not permitted by statutory regulation or exceeds the permitted use, you will need to obtain permission directly from the copyright holder. To view a copy of this license, visit http://creativecommons.org/ licenses/by/4.0/.

(C) The Author(s) 2020 